\title{
Contatto e relazione: dai Touchpoint di Brazelton al massaggio infantile AIMI. Linee di pensiero tra T.B. Brazelton e Vimala McClure ai tempi del Covid-19
}

\author{
Elisabetta Ciracò*, Danila Grande** \\ * Psicologa, psicoterapeuta, insegnante AIMI, già professore a contratto negli ambiti di psicologia clinica e psicologia \\ scolastica, Università di Bologna, sede di Cesena, Dipartimento di psicologia; ** Insegnante AIMI, educatrice nido, Roma
}

\begin{abstract}
I due autori, entrambi attenti al sostegno della genitorialità e allo sviluppo del bambino, non solo si incontrano nelle linee di vedute teoriche e operative, evidenziate negli otto punti in comune, ma ci dimostrano quanto le loro idee sono oggi più che mai attuali. Viviamo in un tempo dove l'azione spesso precede il pensiero e siamo chiamati ad adattarci e rispondere alle aspettative degli altri su come dovremmo essere. Sappiamo quanto questo sia controproducente nella crescita dei figli. Questo articolo propone riflessioni, attenzioni e sguardi a chi si occupa di sostegno alle famiglie e di relazione operatori-bambini nei primi 1000 giorni di vita. La riflessione proposta riconduce verso linee di comunità educante con capacità di ascolto, tempo, empatia e abilità sociali per il benessere dei bambini e delle famiglie. Queste linee sono in sincronia con le attuali indicazioni emerse nelle ricerche scientifiche, nell'ambito dell'epigenetica e delle neuroscienze.
\end{abstract}

The two authors, both attentive to parenting support and child development, not only meet in the theoretical and operational lines of views, highlighted in the eight points in common, but show us how their ideas are today more current than ever. We live in a time where action often precedes thought and we are called to adapt and respond to the expectations of others on how we should be. We know how counterproductive this is in raising children. This paper offers reflections, attentions and views to those involved in supporting families and the relationship between professionals and children in the first 1000 days of life. The proposed reflection leads back to lines of an educating community with listening skills, time, empathy and social skills for the well-being of children and families. These lines are in sync with the current indications that have emerged in scientific research, in the field of epigenetics and neuroscience.

\section{"Quando rafforziamo le famiglie in ultima analisi rafforziamo la comunità. Il nostro obiettivo è che i genitori siano ovunque affiancati da operatori capaci di dare loro sostegno, sviluppino fiducia nelle proprie capacità genitoriali e che formino dei legami di attaccamento forti e resilienti con i propri bambini." \\ T.B. Brazelton, Learning to Listen. A LIFE CARING FOR CHILDREN, 2013}

\section{Introduzione}

Negli ultimi anni la ricerca nel campo della biologia molecolare e, in particolare, dell'epigenetica ha delineato un nuovo modello di genoma dinamico e interattivo con l'ambiente, inteso come fonte di informazioni e sollecitazioni in grado di indurre modifiche fenotipiche reattive e adattative, soprattutto nelle prime fasi della vita. $\mathrm{Su}$ queste basi si va delineando un nuovo modello patogenetico per le malattie croniche $\mathrm{e}$ in particolare per alcuni disturbi del neurosviluppo, che insorgerebbero co- me conseguenza di alterazioni della programmazione epigenetica adattativa a situazioni ambientali che l'embrione/feto percepisce come non favorevoli alla realizzazione del proprio programma genetico. È la tematica della teoria dell'origine fetale delle malattie che ha origine dall'"ipotesi di Barker" e il suo team di Southampton risalente agli inizi degli anni Ottanta (DOHaD, Developmental Origins of $\mathrm{He}-$ alth and Disease). Appare evidente come il modello patogenetico, che impone una riflessione sulle strategie di prevenzione, mira alla riduzione dell'esposizione a fattori di rischio durante la vita embrio-fetale e nella primissima infanzia [1]. Queste recenti ricerche ci riportano al cambio di prospettiva che Brazelton introdusse nella visione del neonato dagli anni Cinquanta in poi. Lo psicologo Barry Lester, nel descrivere il lavoro di Brazelton ha scritto [2]: "Brazelton ha messo il bambino al centro dell'universo della scienza dello sviluppo infantile e ha rivoluzionato il mo- do in cui pensiamo, comprendiamo e studiamo i bambini”. Le intuizioni, lo sguardo e le osservazioni di Brazelton hanno contribuito alla comprensione e visione completamente nuova dei neonati sul loro comportamento, sul loro temperamento, sulle loro interazioni con la famiglia e altri caregiver e la nuova consapevolezza della complessità e delle influenze reciproche dello sviluppo umano. Le scoperte e le idee di Brazelton, molte delle quali sono ora pienamente integrate nella ricerca e nella pratica tradizionali, erano spesso in anticipo sui tempi e si trovavano di fronte all'opposizione dell'establishment medico. T.B. Brazelton, MD, professore emerito, pediatra e psichiatra infantile presso la Harvard Medical School, è stato uno dei più influenti scienziati clinici e sostenitori della pediatria e dello sviluppo infantile del ventesimo secolo. Nel tentativo di raggiungere più bambini e famiglie, e di continuare a influenzare la ricerca, la pratica e la politica, Brazelton nel 1995 riunì a Bruxelles un gruppo di studiosi e professionisti per promuovere la fondazione nel mondo di centri che diffondessero l'insegnamento dell'Approccio Brazelton con trainer specificamente formati a Boston. Esistono diversi centri internazionali. In Italia la "Formazione Brazelton" è presente al Meyer di Firenze dal 1996; la formazione Touchpoints fa riferimento al centro Touchpoints dell'Associazione NATINSIEME di Roma.

Spostandoci dalla relazione al contat to troviamo il grande contributo di Vimala McClure sull'importanza e la forza del massaggio infantile come linguaggio e mezzo comunicativo nell'incontro e sintonizzazione della relazione madre-padre-bambino. Fondatrice dell'International Association of Infant Massage, da decenni si è dedicata a diffondere la cultura del contatto e la pratica del massaggio infantile nei Paesi occidentali. Dopo aver osservato i benefici del massaggio sui neonati durante un viaggio in India, ha voluto studiare 
e conoscere a fondo gli effetti positivi del contat to attraverso la pelle, per creare una sequenza di massaggi facile da apprendere e ricca di benefici per i bambini, per i genitori, per tutta la famiglia. Scrive McClure: "I bambini che sono toccati, tenuti in braccio e massaggiati crescono diventando persone amorevoli, generose e buoni genitori... ho avviato l'associazione internazionale di massaggio infantile con una conoscenza nel mio cuore, l'osservazione dei bambini indiani, e una mole di ricerche fatte sugli animali. Ashley Montagu [3] mi ha consentito di apprendere che tutti i mammiferi sono uguali, e questo rende ragionevole che la ricerca sugli animali possa valere anche per gli umani. Adesso, quarant'anni dopo, vedo le mie idee avverarsi. Il lavoro della mia vita è un miracolo, realizzatosi grazie a persone splendide, che hanno creduto nella verità della mia visione" [4]. Originaria del Colorado, Vimala McClure apprende quest'arte durante un periodo di lavoro in un orfanotrofio in India; nel 1976, tornata negli Stati Uniti e in attesa del primo figlio, decide di studiare e approfondire questa pratica, perfezionandola e integrandola con altre tradizioni esistenti (massaggio indiano e svedese, riflessologia, yoga). Dopo la pubblicazione del suo primo libro, Infant massage: a handbook for loving parents, nel 1981 assieme ad Audrey Downes avvia l'International Association of Infant Massage e la pratica del massaggio infantile inizia a diffondersi uniformemente nel mondo. Nel 1989 nasce l'Associazione Italiana Massaggio Infantile (AIMI) e vengono formati i primi insegnanti. Benedetta $\mathrm{Co}_{-}$ sta, terapista della riabilitazione nel campo pediatrico, che nel 1983 negli Stati Uniti partecipa ai corsi per instructors tenuti da Vimala, al ritorno in Italia introduce l'insegnamento del massaggio presso il servizio di fisioterapia, nell'unità di terapia intensiva neonatale dell'ospedale Gaslini di Genova. I corsi AIMI si tengono in Italia regolarmente dal 1990. Il 6 aprile del 2019 a Bologna si è svolta l'assemblea nazionale dei 30 anni dell'AIMI in Italia. L'AIMI fa parte dell'International Association Infant Massage (IAIM), l'organizzazione che riunisce le varie associazioni nazionali e che ha sede in Svezia dal 1994.

Brazelton e McClure hanno sviluppato preziosi contributi per gli operatori che si occupano di relazione d'aiuto: in ambito educativo, nella scuola primaria (nidi e scuola infanzia) e nei centri per le famiglie (sedi di accoglienza e sostegno alla genitorialità): in ambito sanitario, sia in ospedale che sul territorio, in particolare nei consultori familiari. Accompagnando lo sviluppo dei bambini con la promozione di una comunità educante a supporto del benessere e del rispetto dei valori umani.

Ci siamo proposti di mettere a confronto più aspetti dei contributi dei due autori, quali le diverse vision, i rispettivi focus, per rilevare cosa apportano in sinergia, le rispettive idee sullo sviluppo del bambino, la centralità rispetto alla modalità dell'operatore e alla relazione e infine gli otto principi Touchpoints in chiave AIMI.

I due autori mostrano molte linee di pensiero comuni nella valorizzazione del supporto nella pratica educativa, psicologica e sociale nell'ambito delle cure primarie.

\section{La vision di Brazelton}

Rilevanti sono le descrizioni riportate dal pediatra e neonatologo Gherardo Rapisardi, oltre che formatore nell'Approccio Brazelton, che afferma: "Le differenze individuali, le prime interazioni con i caregiver, la guida anticipatoria, l'assistenza centrata sulla famiglia, la teoria dei sistemi dinamici nello sviluppo umano e la collaborazione interdisciplinare sono solo alcuni dei campi di studio più diffusi e noti in cui il lavoro di Brazelton continua a influenzare la ricerca, la pratica clinica e i piani d'azione. Fin dagli anni '50 Brazelton ha dato un forte impulso al riconoscimento delle competenze del neonato, visto come un essere sociale, predisposto a interagire attivamente con la madre e con le altre persone che si prendono cura di lui, riuscendo a influenzare il tipo di accudimento necessario alla propria sopravvivenza. [...] Chi ha conosciuto personalmente Berry Brazelton sa che la capacità di leggere il comportamento del bambino era parte integrante della sua personalità. Vederlo al lavoro con i bambini era affascinante per tutti, dai genitori che si sentivano non giudicati ma sostenuti con empatia e da una guida sicura, ai bambini facilmente catturati da un grande uomo simpatico che usava con naturalezza il loro linguaggio, agli operatori che si riconoscono nelle parole di Daniel Stern: guardandolo viene voglia di saper fare ciò che lui fa con il bambino, solo in parte connesso con il sapere e molto più vicino al saper essere" [5].

In uno dei suoi ultimi libri Brazelton racconta uno dei passaggi fondamentali: " $\mathrm{Co}$ minciai a pensare che il mio lavoro consisteva nell'imparare ad apprezzare i genitori. Quando cambiai il mio atteggiamento e iniziai a fidarmi di loro, loro cominciarono ad avere più fiducia in sé stessi” [6].

\section{Approccio Brazelton}

Il focus sull'uso del linguaggio del comportamento del bambino come mezzo per entrare in relazione con i genitori.
Tra gli aspetti più creativi e originali del suo contributo ritroviamo il riconoscimento del linguaggio del comportamento del bambino come mezzo dell'operatore per entrare in relazione con i genitori e sostenerne le competenze. Questo determina l'apertura di un canale privilegiato nella comunicazione con loro.

Nell'approccio Touchpoints, che prende il nome dai momenti sensibili dello sviluppo, si ritrovano gli aspetti che offrono l'opportunità di avvicinarsi al "sistema famiglia" in periodi in cui il comportamento del bambino si disorganizza e, di conseguenza, può disorientare i genitori, alle prese con i loro compiti di accudimento e definizione dei propri stili educativi.

Nello specifico, i Touchpoints sono dei periodi prevedibili di regressione e disorganizzazione che precedono dei salti in avanti nello sviluppo del bambino, spesso accompagnati da frustrazione e dubbi da parte dei genitori. Questi periodi di disorganizzazione possono mettere in crisi le relazioni familiari, ma possono anche costituire un'opportunità per gli operatori per entrare in contatto e collaborare con i genitori. Riuscire a leggere e comprenderne significati e segni è parte integrante della rete di sostegno di cui i nuovi genitori sembrano avere sempre più bisogno a oggi. Essere aiutati a comprendere i comportamenti del bambino, le caratteristiche della fase del suo sviluppo e allo stesso tempo i propri istinti, emozioni, frustrazioni e disorientamenti verso di lui favorisce l'entrare in contatto con il mondo della crescita e dello sviluppo infantile, aumentando la confidenza e il senso di competenza che si prova in questo difficile ruolo, soprattutto nei primi anni di vita.

Gli aspetti essenziali dell'approccio Brazelton fanno riferimento ai bisogni irrinunciabili dei bambini (Tabella 1), con gli obiettivi di sostenere i genitori e di promuovere le fasi critiche e fisiologiche dello sviluppo dei bambini [7].

I pilastri concettuali su cui si basa l'approccio sono costituiti da una vasta gamma di conoscenze sullo sviluppo del bambino e del genitore, affiancate dalla comprensione di come la pratica clinica si inserisce nella relazione umana. I principi della pratica sono degli strumenti da utilizzare nel lavoro quotidiano con le famiglie e aiutano l'operatore a tenere ben a mente gli elementi essenziali dell'approccio Touchpoints (Tabella 2).

Tra i principali obiettivi dell'approccio Touchpoints vi sono:

- il sostegno dei genitori e promozione delle loro competenze (autostima, sicurezza e coerenza); 
TABELLA 1. L'approccio Brazelton sullo sviluppo del bambino

I bambini hanno il bisogno irrinunciabile:

di sviluppare costanti relazioni di accudimento;

di protezione fisica, sicurezza e relativa normativa;

di esperienze modellate sulle differenze individuali;

di esperienze adeguate alla fase di sviluppo; - di definire dei limiti, di fornire una struttura e delle aspettative;

di comunità stabili e di supporto e di continuità culturale;

di salvaguardare il futuro.

TABELLA 2. L'approccio Brazelton per l'operatore

- Valorizza e comprendi la relazione tra te e il genitore.

Utilizza il comportamento del bambino come tuo linguaggio.

Riconosci ciò che apporti all'interazione.

Sii disposto a discutere di argomenti che vanno oltre il tuo ruolo tradizionale.

Cerca le opportunità per sostenere le competenze genitoriali.

Focalizzati sulla relazione genitore bambino. - Valorizza la passione ovunque tu la trovi.

- Considera la disorganizzazione e la vulnerabilità come opportunità.

- la promozione della salute psicomotoria del bambino prevenendo possibili disturbi relazionali.

Diventa così prioritario poter collaborare con i genitori per creare una comprensione comune del bambino per aiutarlo a sviluppare fiducia in sé, regolare il proprio comportamento e le proprie emozioni. Sapere di cosa hanno bisogno i bambini per un'armonica crescita evolutiva e sociale.

\section{La vision di Vimala McClure}

"Credo che diffondendo e incoraggiando l'infant massage e le altre tradizioni culturali che accrescono il rapporto affettivo tra genitori e bambini [...] cominceremo a vedere intere generazioni esprimere più amore e responsabilità per i loro simili. Credo che i bambini siano esseri umani consapevoli che meritano rispetto, tenerezza e calore e, soprattutto, un cuore che ascolta. Io credo che ogni genitore, a prescindere dalla sua filosofia personale, e ogni bambino, a prescindere dalla storia della sua nascita o dalla sua predisposizione, dovrebbero avere l'opportunità di sperimentare i benefici che durano tutta la vita e che derivano dai primi legami costruiti dall'amore, dalla salute e dalla sicurezza" (Vimala Schneider McClure, Infant Massage Instructor's Manual, 1979).

\section{Il senso del massaggio}

Il massaggio infantile non è una tecnica, ma un modo di stare con il proprio bambino. È un'antica tradizione, dove il contatto pelle a pelle rappresenta il mezzo privilegiato con cui il genitore trasmette al bambino amore, affetto, ascolto e rispetto. Subito dopo la nascita, il bambino trae un prezioso nutrimento dal contatto con i genitori, dai loro sorrisi, dai loro sguardi affettuosi, dalle loro amorevoli parole, dalla sintonizzazione della relazione. Il massaggio diventa così un momento speciale di comunicazione profonda. Attraverso il contatto, i genitori ascoltano i bisogni del neonato, agganciano lo sguardo, imparano a comprenderli e a rispondere in modo adeguato. Negli ultimi anni si è sviluppato un nuovo filone di ricerca scientifica, focalizzato proprio sullo studio del rapporto tra la neurobiologia sistemica e le emozioni nel sistema uomo [8]. Le ricerche di neuroimmagini sono infatti in grado di mostrarci la sinfonia dei nostri straordinari circuiti: flussi sincronici di qualità distinte, pensieri, emozioni, sentimenti in un continuo scorrere l'uno nell'altro [9], Sappiamo, come dimostrato da studi sul campo, che degli interruttori che accendono le emozioni positive "pro sociali" sono certamente l'abbraccio e il contatto fisico. L'ormone della relazione, l'ossitocina, ha un ruolo determinante nel comportamento materno, nella creazione di un legame d'affetto tra le persone, specialmente tra mamma e il suo bambino, e nei rapporti sociali. Sembra essere anche collegata ai sentimenti di fiducia tra gli esseri umani così pure a ridurre lo stress, limitando l'ansia sociale permette la costruzione di relazioni migliori. Anche il sorriso, la carezza e il tocco sono degli interruttori emozionali. Fondamentali per un neonato sono l'abbraccio, la carezza e il contatto fisico perché sono i gesti più antichi dell'accudimento, del dare e del ricevere affetto. $\mathrm{Ci}$ fanno sentire bene e siamo programmati per andare a cercarli [10]. Nell'esperienza del massaggio infantile, i genitori e il bambino, sperimentano tutti questi elementi essenziali, fondamenta di base per una buona relazione umana!

\section{Cosa ritroviamo in comune \\ tra i due autori}

In entrambi gli autori si intersecano vedute e idee sia in merito alla concezione dello sviluppo del bambino che negli aspetti prettamente operativi della relazione d'aiuto, tra operatore e famiglie per il benessere del sistema familiare stesso.

Entrambi rivolgono lo sguardo al modello evolutivo concordando, in particolare, su:
- sviluppo discontinuo e in parallelo;

- relazione genitore-bambino come processo.

Sintonia di vedute sul modello relazionale:

- il focus è sull'alleanza che si crea fra operatore e genitore;

- l'operatore è un elemento del sistema che sostiene la crescita.

Qui di seguito riportiamo gli aspetti principali della proposta di McClure all'interno degli otto principi guida dell'approccio Touchpoints di Brazelton:

1 Valorizza e comprendi la relazione fra te e il genitore. Nelle linee AIMI è solo il genitore che entra in contatto pelle a pelle con il bambino, perché è lui il miglior esperto del bambino, valorizza le sue competenze sostenendo anche il suo benessere personale (rompighiaccio) e ponendosi in ascolto rispetto ad ansie e preoccupazioni. Operatore $\Leftrightarrow$ genitore in partnership.

2 Utilizza il comportamento del bambino come tuo linguaggio. Il comportamento del bambino diviene per i caregiver il modo di "parlare la sua stessa lingua”. L'osservazione dei segnali in termini di disponibilità o indisponibilità comportamentali del bambino attraverso: il contatto visivo; i segnali del viso; i suoni emessi e il linguaggio del corpo aiutano i genitori a dare valore all'ascolto attivo e confermano il nutrimento affettivo familiare che risponde al bisogno di sviluppare costanti relazioni di cura.

3 Valorizza la passione ovunque la trovi. La passione che i genitori apportano alla relazione include ambivalenza di sentimenti (Brazelton). Il massaggio li "mette in gioco" anche nel "tirar fuori” rabbia, confusione e paura di non farcela, di non essere all'altezza. Forma inoltre l'operatore in un costante esercizio di osservazione empatica del proprio sentire verso la coppia genitoriale.

4 Focalizzati sulla relazione genitore-bambino. Dalla relazione col genitore il bambino trae nutrimento e stimolo verso nuove richieste di cura. Il corso di massaggio si focalizza sulla relazione come un'occasione privilegiata per comprendersi, sperimentare che amare non è viziare e che questa esperienza li fa nascere come genitori e persone nuove.

5 Cerca le opportunità per sostenere le competenze genitoriali. Nell'approccio AIMI il massaggio punta sullo sviluppo del bambino e sull'incontro e sin- 
tonia tra genitori e bambino. L'impostazione olistica non soltanto sostiene la sfida genitoriale, ma ne comprende l'aspetto sociale nell'incontro con altre coppie e vissuti simili. L'operatore si pone come modello di riferimento nella relazione di cura.

6 Riconosci ciò che apporti alla relazione. I caregiver sono chiamati a vivere la relazione come sfida di crescita anche con loro stessi e con le loro personali "disorganizzazioni". Se è vero che per "insegnare" bisogna imparare, grande risalto deve avere il "vissuto interiore", accogliere i sentimenti di ambivalenza, consentendo di affrontarli con maturità e formazione professionale.

7 Sii disposto a discutere argomenti oltre il tuo ruolo. Vimala McClure individua proprio nella mission la "cura dell'altro" a partire dalla "cura di sé". Brazelton sottolinea la necessità del confronto, dell'apertura agli altri operatori (fare rete per promuovere il ruolo) per affinare le capacità di leggersi durante l'interazione in un processo di sperimentazione continua.

8 Considera la disorganizzazione e la vulnerabilità come opportunità. Fasi di disorganizzazione, seguite da con- solidamento, rappresentano un aspetto naturale dello sviluppo.

\section{Conclusioni}

I due autori, entrambi attenti al sostegno della genitorialità e allo sviluppo del bambino, non solo si incontrano nelle linee di vedute teoriche e operative, evidenziate negli otto punti in comune, ma ci dimostrano quanto le loro idee sono oggi più che mai attuali. Soprattutto in questi giorni dopo il lungo periodo di confinamento e cambio sociale e di vita imposto dalla pandemia del Covid-19 che ha messo in discussione l'equilibrio delle sicurezze di base. Nonostante il distanziamento introdotto per ridurre il contagio, non possiamo dimenticare le leggi non scritte della nostra società, la vicinanza fisica è conferma di conforto e sostegno e fondamentale se si parla di bambini. Gli autori ci sono di aiuto nel sottolineare ciò che conferma la letteratura scientifica, sia in ambito epigenetico che delle neuroscienze, rispetto all'importanza del contatto e della relazione in tutto il cammino della nostra vita. Sarà fondamentale il lavoro di ricerca delle conseguenze sui bambini di questo periodo di distanziamento e di riduzione dei contatti per "salvaguardare il futuro", in stretta collaborazione tra famiglie e operatori, e far seguire alla "fase di disorganizzazione" di questi mesi un grande consolidamento che riconduca l'esperienza vissuta a un "aspetto naturale dello sviluppo".

\luisaciraco@gmail.com

1. Burgio E. Dalla genetica all'Epigenetica. Soma Istituto Milano, 2019

2. Lester B, Sparrow J. Bambini e famiglie. L'eredità di T. Berry Brazelton. Edizione italiana cura di Migliaccio L, Rapisardi G. Raffaello Cortina editore, 2015.

3. Montagu A. Il linguaggio della pelle. Verdechiaro edizioni, 2018.

4. Schneider McClure V. Infant Massage Instructor's Manual. 1979.

5. Rapisardi G. Berry Brazelton. Quaderni acp 2018;3:98.

6. Brazelton TB. Learning to Listen. A Life Caring for Children. Da Capo Press, 2013.

7. Brazelton TB, Greenspan SI. I bisogni irrinunciabili dei bambini. Raffaello Cortina editore, 2001.

8. Mc Gaugh JL. Consolidating memories. Annu Rev Psychol. 2015 Jan 3;66:1-24.

9. Della Sala S. Le neuroscienze a scuola. I1 buono, il brutto, il cattivo. Giunti, 2016

10. Lucangeli D. Cinque lezioni leggere sull'emozione di apprendere. Centro Studi Erickson, 2019

\section{La cultura del dolore}

II dolore è una percezione complessa, multidimensionale, che varia in qualità, forza, durata, ubicazione e spiacevolezza. L'esperienza del dolore non si riduce alla descrizione di uno stimolo nocicettivo che viene trasmesso dalle vie nervose periferiche al sistema nervoso centrale; fattori esterni alla persona, come la famiglia, il vivere sociale e la cultura regolano alcune dimensioni della percezione del dolore e, soprattutto, della sua comprensione.

Ed è su questa visione della biologia del dolore umano che alcuni ricercatori del Regno Unito e del Canada hanno analizzato il modo in cui sono state rappresentate le esperienza di dolore dei personaggi animati che i bambini guardano alla televisione. Analizzando 6 serie televisive e 10 film per bambini e famiglie rivolti soprattutto a bambini di 4-6 anni, per un totale di 52 ore di video, sono stati esaminati gli incidenti che causavano il dolore a cui erano esposti i personaggi, con un'analisi dell'evento doloroso e della reazione delle vittime e dei personaggi intorno a loro. Sono stati riconosciuti 454 eventi in cui era rappresentato il dolore con una media di 8,66 episodi di dolore/ora, con il dolore a insorgenza acuta - incidente improvviso - quello più comunemente rappresentato (79\%). I personaggi maschili hanno avuto più probabilità di provare un dolore intenso, così come decodificato dalle espressioni facciali, mentre i dolori che occorrono più comunemente al bambino, come per esempio la sbucciatura di un ginocchio a causa di una caduta, erano scarsamente rappresentati ( $20 \%$ del totale degli incidenti). Un aspetto interessante della ricerca è l'analisi dei sentimenti vissuti dai personaggi che hanno assistito all'evento: nel $41 \%$ dei casi coloro che erano presenti non hanno avuto manifestazioni di empatia con la vittima 0 , addirittura, si sono dimostrati indifferenti [1]. Se è vero che la televisione influenza i comportamenti dei bambini, questi programmi dedicati ai bambini potrebbero fare molto di più per rappresentare meglio l'esperienza di dolore vissuta realmente dai bambini, dal dolore cronico a quello quotidiano, riducendo le raffigurazioni del dolore violento o estremo, e mostrando più empatia quando i personaggi mostrano dolore. "Ma quando nel dolore si hanno compagni che lo condividono, l'animo può superare molte sofferenze", così fa dire a Edgar il sommo Shakespeare, ma oggi questa del Re Lear non sembra una narrazione di successo.

1. Mueri K, Kennedy M, Pavlova M, et al. The sociocultural context of pediatric pain: an examination of the portrayal of pain in children's popular media. Pain. 2021 Mar 1;162(3):967-75. 